\title{
Liter per Day per Microgram per Kilogram per Day
}

National Cancer Institute

\section{Source}

National Cancer Institute. Liter per Day per Microgram per Kilogram per Day. NCI

Thesaurus. Code C120735.

A unit of concentration equal to liter per day divided by microgram per kilogram per day. 